\title{
Axial Magnetic Field Effects on a Saturated He-Ne Laser Amplifier
}

\author{
ROBERT T. MENZIES, ANDREW DIENES, AND NICHOLAS GEORGE, SENIOR MEMBER, IEEE
}

\begin{abstract}
The behavior of a He-Ne laser amplifier in the presence of an axial magnetic field has been studied experimentally by measuring Faraday rotation and gain for various values of input signal intensity. Two high-gain transitions in the 3.39- $\mu$ region were used for study-a $J=1$ to $J=2$ transition and a $J=1$ to $J=1$ transition. Theoretical expressions have been developed, which included the nonlinear effects of saturation strength signals. Experimental results clearly show saturation of Faraday rotation; in addition, for the $J=1$ to $J=1$ transition, a Faraday rotation reversal and a traveling-wave magnetic field dip are seen. These results match the theoretical predictions and provide a method for measuring the upper $\left(3 s_{2}\right)$ level quadrupole moment decay rate.
\end{abstract}

\section{INTRODUCTION}

M ANY authors have discussed the nonlinear interactions of electromagnetic fields with a gaseous laser medium in the presence of a magnetic field, which splits the degenerate atomic levels. Recently Sargent, Lamb, and Fork [1] presented a comprehensive theory of a Zeeman laser oscillator based on the calculation of the induced polarization in the gaseous medium. The polarization was calculated in the form of a truncated perturbation series, valid for the field strengths existing in a laser that is oscillating near threshold. Many predictions of this theory were supported by the experimental work done by Tomlinson and Fork [2] with singlemode $\mathrm{He}-\mathrm{Ne}$ Zeeman lasers operating on transitions at $632.8 \mathrm{~nm}$ and $1.52 \mu$.

Dienes has analyzed the behavior of a saturated gaslaser amplifier in the presence of an axial magnetic field. The weak-signal theory [3] is based on a perturbation series method similar to that of [2], and the strong-signal theory [4] is based on an exact integration of the equations of motion for the macroscopic density matrix. In this analysis the input signal consists of two fields of arbitrary polarization, and a study is made of the effects of the gaseous medium on the signal. In most cases the angular momenta of the energy levels involved in the laser trans-

Manuscript received June 30, 1969; revised September 8, 1969. The work of R. T. Menzies and N. George was supported in part by the Electronics Division of the Air Force Office of Scientific Research. This paper is based in part on partial fulfillment of the Ph.D. degree, California Institute of Technology, Pasadena, Calif., by R. T. Menzies.

R. T. Menzies and N. George are with the California Institute of Technology, Pasadena, Calif.

A. Dienes was with the California Institute of Technology, Pasadena, Calif. He is now with Bell Telephone Laboratories, Inc., Holmdel, N. J. ition play a large part in the effect of the medium on the laser signal.

In this paper we report the results of an investigation of the effects of a $\mathrm{He}-\mathrm{Ne}$ Zeeman laser amplifier on signals in the vicinity of two high-gain transitions: the $3 s_{2}-3 p_{4}$ $(J=1$ to $J=2$ ) neon transition at $3.3923 \mu$, and the $3 s_{2}-3 p_{2}(J=1$ to $J=1)$ neon transition at $3.3912 \mu$ [5], [6]. Theoretical expressions have been derived which describe the Faraday rotation and amplification of a linearly polarized signal as a function of axial magnetic field. These expressions involve several atomic parameters, and comparisons of experimental measurements with these predictions yield useful information about the properties of the medium, without involving the complicating effects of an oscillator cavity. The transitions that were chosen for study have high gain and easily saturate the laser medium. They normally operate under strongly saturated conditions in a laser oscillator, and this makes a comparison of experiment with the perturbation theory of a Zeeman-laser oscillator [1] rather difficult. However these attributes are conducive to a study using a laser amplifier, where the input signal strength can be varied and treated as an external parameter.

\section{Theory and its Application to the 3.39- $\mu$ Lines}

In this section we describe the pertinent theory and present results of both the perturbational and strongsignal methods.

When considering the treatment of an amplifier operating on a single-frequency signal, the appropriate assumption is that the field amplitudes are purely space-dependent. Thus we start with (28a) and (28b) of [3] and set time derivatives equal to zero to obtain the gain and phase equations.

$$
\begin{aligned}
E_{m} \frac{d \phi_{m}}{d z} & =\frac{\nu}{2 \epsilon_{0} c} P_{c m} \\
\frac{d E_{m}}{d z} & =\frac{\nu}{2 \epsilon_{0} c} P_{s m}
\end{aligned}
$$

where the electromagnetic field is $E=\operatorname{Re} \sum_{m} \hat{e}_{m} E_{m} \exp$ $\left(i k z-i \nu t+i \phi_{m}\right), P_{m}=P_{c m}-i P_{s m}$ is the complex amplitude of the induced polarization, and $m$ is + or denoting right or left circular polarization state.

According to the third-order perturbational theory for a general $J_{a}$ to $J_{b}$ transition in a laser amplifier subject to an axial magnetic field, the left circular component of the 
induced polarization due to a single-frequeney signal tuned to line center is

$$
\begin{aligned}
\left(\nu / 2 \epsilon_{0} c\right) P_{-}=- & i \alpha E_{-} \sum_{M} C_{M, M-1}^{2}\left\{w^{*}\left(y^{b}+M \delta+i a\right)\right. \\
& -\left(E_{-}^{2} / 2 E_{0}^{2}\right) C_{M, M-1}^{2} H_{1 M} \\
& -\left(E_{+}^{2} / 2 E_{0}^{2}\right)\left[C_{M-2, M-1}^{2}\left(H_{6 M}^{a}+H_{7 M}^{a}\right)\right. \\
& \left.\left.+C_{M, M+1}^{2}\left(H_{6 M}^{b}+H_{7 M}^{b}\right)\right]\right\} .
\end{aligned}
$$

This expression and the corresponding expression for the right circular component of the induced polarization can be deduced from (36) and subsequent comments in [3]; here, as in that paper, $a=\gamma_{a b} / k u, A=\gamma_{a} / 2 k u, B=$ $\gamma_{b} / 2 k u, y^{a}=\omega_{+-}^{a} / 2 k u, y^{b}=\omega_{+-}^{b} / 2 k u, \delta=y^{a}-y^{b}$, where $\gamma_{a b}$ is the natural linewidth, $\gamma_{a}$ and $\gamma_{b}$ are the decay rates of the upper and lower levels $(a=A+B$, since collision effects are neglected), $\omega_{+-}^{a}$ and $\omega_{+-}^{b}$ are the Zeeman splittings of the upper and lower levels $\left(\omega_{+-}^{i}=g_{i} e B / m\right)$, and $k u$ is the Doppler width parameter which is used to normalize the above quantities $\left(k u=(\pi / \sqrt{\ln 2}) \Delta \nu_{D}\right.$ where $\Delta \nu_{D}$ is the full width at half maximum of the Doppler broadened gain curve). $\alpha$ is the small-signal inhomogeneous gain constant, $E_{0}^{2}=3 h^{2} \gamma_{a} \gamma_{b} /|\langle a\|p\| b\rangle|^{2}$ is the saturation field intensity, and $C_{M, M}^{2}=\left[3 /\left(2 J_{b}+1\right)\right]^{2}$ $\left[<J_{a}, 1, M,-1\left|J_{b}, M^{\prime}\right\rangle\right]^{2}$, with the summation running over all $M$ values of the upper level. $H_{1 M}, H_{6 M}$, and $H_{7 M}$ are complicated functions describing the nonlinear polarization and depend on the parameters $a, A$, and $B$, as well as on the magnetic field. The function $w(z)$ is the error function of complex argument [7] which describes the dependence of the first-order (linear) polarization of the medium on the atomic parameters and on the magnetic field.

The gain and Faraday rotation of a linearly polarized signal, where $E_{+}=E_{-}=E / \sqrt{2}$, can then be expressed as

$$
\begin{gathered}
(1 / I) d I / d z=2 \alpha \operatorname{Re}[W(y)-I F(y)], \\
d \Phi / d z=\alpha \operatorname{Im}[W(y)-I F(y)],
\end{gathered}
$$

where

$$
\begin{aligned}
& W(y)=\sum_{M} C_{M, M-1}^{2} w^{*}\left(y^{b}+M \delta+i a\right), \\
& \begin{aligned}
F(y)=\frac{1}{4} \sum_{M A} C_{M, M-1}^{2}\left[C_{M, M-1}^{2} H_{1 M}\right. \\
+ \\
+C_{M-2, M-1}^{2}\left(H_{6 M}^{a}+H_{7 M}^{a}\right) \\
\left.+C_{M, M+1}^{2}\left(H_{G M}^{b}+H_{7 M}^{b}\right)\right],
\end{aligned}
\end{aligned}
$$

$I=E^{2} / E_{0}^{2}, \Phi=\left(\phi_{-}-\phi_{+}\right) / 2$, and Re and Im denote the real and imaginary parts, respectively. These differential equations can be integrated to yield expressions suitable for comparison with experimental results:

$$
I(z)=\frac{I_{0} \exp (2 \alpha \operatorname{Re} W(y) z)}{1+\left[I_{0} \operatorname{Re} F(y) / \operatorname{Re} W(y)\right][\exp (2 \alpha \operatorname{Re} W(y) z)-1]}
$$

$$
\begin{aligned}
\Phi(z)=\alpha\{\operatorname{Im} W(y) z & -[\operatorname{Im} F(y) / \operatorname{Re} F(y)][\operatorname{Re} W(y) z \\
+ & \frac{1}{2 \alpha} \ln \mid I_{0} \operatorname{Re} F(y) / \operatorname{Re} W(y) \\
+ & \left(1-I_{0} \operatorname{Re} F(y) / \operatorname{Re} W(y)\right) \\
& \cdot \exp (-2 \alpha \operatorname{Re} W(y) z) \mid]\}
\end{aligned}
$$

where $I_{0}=I(z=0)$. These equations result from thirdorder perturbation theory and will remain valid provided $I(z) \ll 1$.

Since for the two laser transitions of interest the difference in the $g$ factors of the levels is small enough $(\delta \simeq$ $\left.0.07 y^{b}\right)$ to be of little significance in these experiments, we neglect $\delta$ and replace $y^{a}$ and $y^{b}$ by $y=\omega_{+-} / 2 k u$. This simplifies formulas considerably since none of the functions now depends on $M$ and they can be removed from the summation signs. The subscript $M$ on the functions will henceforth be omitted. The nonlinear behavior of the gain and Faraday rotation depends on the properties of the saturation functions $H_{1}, H_{6}$, and $H_{7}$. These have been discussed in detail in [4]; only some salient points pertinent to the particular transitions will be mentioned here.

$H_{1}$ describes normal saturation and contributes through the entire Doppler bandwidth. Its interesting feature is that the imaginary part-saturation of the Faraday rotation-is negligible if $a \ll 1\left(\gamma_{u b} \ll k u\right.$, inhomogeneous line). For the transitions considered here $a \simeq 0.3$, i.e., the lines are partially homogeneous. Therefore, we expect a measurable saturation of the Faraday rotation for all values of the magnetic field. Physically, this saturation takes place via power broadening of the natural linewidth of the transition.

$\left(H_{6}+H_{7}\right)$ describes the interaction of the left and right circularly polarized components of the electromagnetic field through a pair of Zeeman split upper (superscript $a$ ) or lower (superscript $b$ ) levels. It can be shown that, because it is due to coherent interactions, $\left(H_{6}+H_{7}\right)$ has a characteristic width equal to the decay rates of the pertinent sublevels. In particular $\operatorname{Im}\left(H_{6}^{a}+\right.$ $\left.H_{7}^{a}\right)$ peaks at $y \simeq A\left(\omega_{+-} \simeq \gamma_{a}\right)$. For the $3.39-\mu$ transitions $A \ll B$ and $a \simeq B\left(\gamma_{a} \ll \gamma_{b}, \gamma_{a b} \simeq \gamma_{b} / 2\right)$. Therefore, since the linear part of the rotation ( $\operatorname{Im} w^{*}$ ) is very small at $y=A$, there will be a dip in the rotation at small values of the magnetic field provided the coefficient of $H_{6}+H_{7}$ is large enough.

For the $J=1$ to $J=1$ transition at $3.3912 \mu$ the coefficient of $H_{6}^{\alpha}+H_{\tau}^{\alpha}$ is $0.25 .{ }^{1}$ Thus, we expect a small, narrow dip in the rotation versus magnetic field curve at low magnetic fields (where $y \simeq A$ ). With increasing signal strength, the dip should increase and a reversal of the rotation might actually occur. A detectable dip in the gain at zero magnetic field should also occur for analogous

\footnotetext{
1 These coefficients are tabulated in [3].
} 
reasons. ${ }^{2}$ The coefficient of $H_{6}^{b}+H_{7}^{b}$ is also 0.25 . It does not produce an anomalous dip, however, since $H_{6}^{b}+H_{7}^{b}$ is much broader, its imaginary part peaking at $y=B$. This part of the nonlinear polarization behaves rather similarly to $H_{1}$ and contributes to saturation of both gain and Faraday rotation through most of the Doppler width.

For the $J=1$ to $J=2$ transition at $3.3923 \mu$, the coefficient of $H_{6}^{a}+H_{7}^{a}$ is 0.01 . Thus the Faraday rotation is not expected to show an anomalous dip, and for analogous reasons we would not expect to see the very small gain dip. The coefficient of $H_{6}^{b}+H_{7}^{b}$, however, is large, and this part of the nonlinear polarization contributes to the saturation for most values of the magnetic field.

As it was pointed out previously, the perturbational equations become quantitatively invalid at high signal strengths. (However, in [4] it has been shown that the perturbational theory correctly predicts all the strongsignal nonlinear phenomena in a qualitative sense.) Two approximate expressions may be obtained from the strongsignal theory [4]. For high magnetic fields (neglecting differences in $g$ factors again)

$$
\begin{gathered}
I^{-1} d I / d z=2 \alpha \sum_{i M} C_{M, M-1}^{2}\left(1+0.5 I C_{M, M-1}^{2}\right)^{-1 / 2} \\
\cdot \operatorname{Re} w^{*}\left(y+i b_{M}\right), \\
d \Phi / d z=\alpha \sum_{M} C_{M, M-1}^{2} \operatorname{Im} w^{*}\left(y+i b_{M}\right),
\end{gathered}
$$

where

$$
b_{M}=a\left(1+0.5 I C_{M, M-1}^{2}\right)^{1 / 2} .
$$

This expression is valid when the Zeeman splitting is much larger than the power-broadened natural linewidth $\left(y \gg b_{M}\right)$. The approximation involved is analogous to neglecting $H_{6}+H_{7}$ in the perturbational expressions. In this approximation the left and right circular components of the electromagnetic field do not interact. Since the natural linewidth is rather large for our transitions, (6) is valid for a limited range of magnetic field only.

For the $J=1$ to $J=2$ transition if we neglect the contribution of $H_{6}^{a}+H_{7}^{a}$ (which, as it was shown before, is very small) and then make the approximation $\gamma_{b} / 2=\gamma_{a b}$ $(B=a)$ the following expression, valid for arbitrary signal strength, may be obtained. ${ }^{3}$

$$
\begin{aligned}
& I^{-1} d I / d z+2 i d \Phi / d z=\frac{2 \alpha}{\pi} \sum_{i=1,2,3} \int_{-\infty}^{+\infty} \\
& \qquad \begin{aligned}
c_{1,2,3} & =0.6,0.1, \text { and } 0.3 \\
d_{1,2,3} & =0.1,0.6 \text {, and } 0.3 .
\end{aligned}
\end{aligned}
$$

2 This is the so-called "traveling-wave magnetic-field dip," which has been previously observed in laser oscillators. See for example [2]. ${ }^{3}$ See (17) of. [4] for the derivation of a similar equation for the $J=1 / 2$ to $J=3 / 2$ transition.
In this approximation the left and right circular components of the electromagnetic field interact via the lower sublevels only. Equations (5), (6), and (7) will be used in Section IV to compare experimental results with theoretical curves. The nonlinear differential equations in (6) and (7) must be integrated numerically. In addition, the rather complicated Doppler integral in (7) must also be numerically evaluated. ${ }^{4}$

\section{EXPERIMENT}

The experimental setup is shown in Fig. 1. The output of a tunable, single $\mathrm{TEM}_{00 \alpha}$ mode, $3.39-\mu \mathrm{He}-\mathrm{Ne}$ laser oscillator with Brewster angle windows was passed through a variable attenuator consisting of two calcite polarizers, collimated with a quartz lens, and passed through an amplifier tube with a discharge length of $50 \mathrm{~cm}$ and a bore diameter of $3.8 \mathrm{~mm}$. The beam diameter was approximately $2 \mathrm{~mm}$ throughout the length of the tube. The amplifier tube was filled to a pressure of 1.0 torr with a 9:1 mixture of $\mathrm{He}^{3}$ and $\mathrm{Ne}^{20}$, and it was centered in a magnetic field solenoid that covered the entire discharge length. The normals of the plane windows of the tube were tilted about $2^{\circ}$ from the axial direction in order to reduce reflection instabilities.

Magnetic field measurements were made using a Bell 120 gaussmeter with an axial probe. Effects of the steel table on the magnetic field produced at the position of the amplifier tube were found to be negligible. The slight inhomogeneities in the axial magnetic field produced by the solenoid produced negligible error compared with experimental accuracy limits due to random instabilities. This was determined by numerically integrating (3a) and (3b) using appropriate sets of input parameters and letting magnetic field strength vary with distance in the axial direction. The results were compared with corresponding results obtained by assuming a uniform average value of field strength. Effects of small residual transverse fields were also studied, and they were found to have a negligible effect on the experimental accuracy.

The amplifier output beam passed through a chopper and was split by a silicon flat whose normal was placed at a small angle with respect to the beam direction. The transmitted portion was analyzed with a calcite analyzer and detected with an InAs photodiode. The reflection 


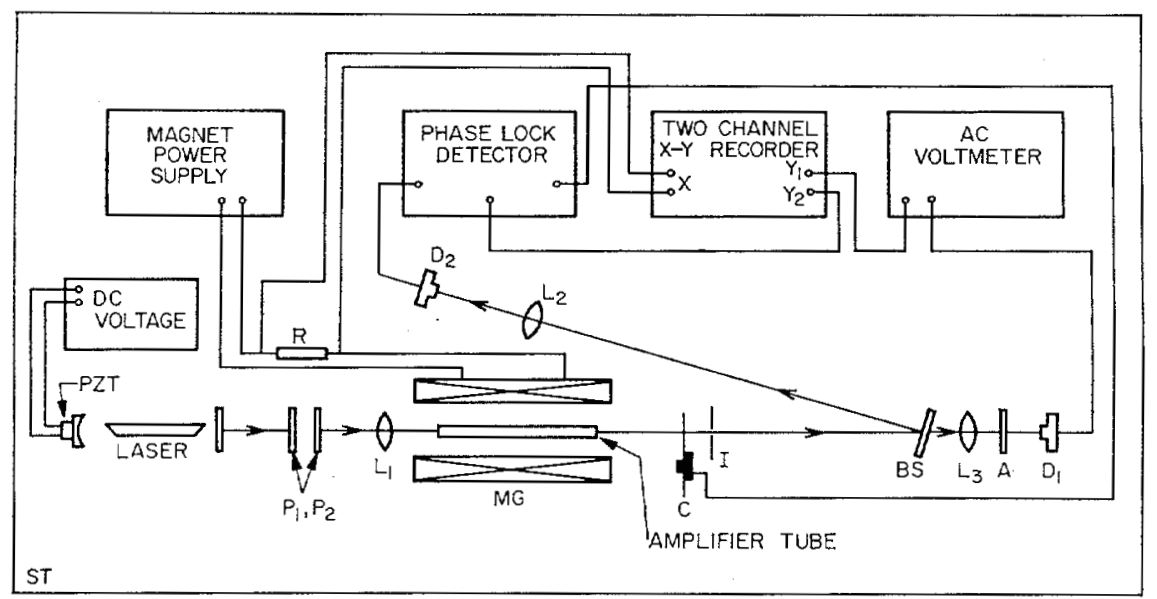

Fig. 1. The experimental setup. $A$-analyzer; $B S$-beam splitter; $C$-chopper; $D_{1}, D_{2}-$-InAs detectors; $I$-iris; $L_{1}, L_{2}, L_{3}$ - lenses; $M G$-magnet; $P_{1}, P_{2}$-attenuating polarizers; $P Z T-$ piezoelectric tuner; $R$-resistor; $S T$-steel table.

field. For the magnetic-field experiments each dc output was connected to a separate $Y$ channel of an $X Y$ twochannel recorder. The $X$-axis deflection was proportional to the axial magnetic field. The analyzer in channel 1 was. set at $45^{\circ}$ with respect to the plane of polarization of the signal when the magnetic field was zero. If we call the signal intensity at zero field $I_{0}$ and the signal intensity at a nonzero value of magnetic field $I(y)$, then the two recorder plots yield the quantities $I(y) \cos ^{2} \theta / I_{0} \cos ^{2}(\pi / 4)$ and $I(y) / I_{0}$. Consequently, the gain variation with magnetic field was obtained directly and the Faraday rotation angle $\Phi=\theta-(\pi / 4)$ was obtained through simple calculations.

The method used to tune the signal to the line center was to pass a weak signal through the amplifier tube in the presence of an axial magnetic field of approximately 70 gauss and tune the oscillator signal to the point at which the ellipticity of the amplifier output signal decreases to zero. The maximum drift encountered during the observation periods was a $\pm 2-\mathrm{MHz}$ deviation from line center. However, the Faraday rotation and gain versus axial magnetic field are not sensitive to deviations from line center of this magnitude; computations based on the perturbational theory equations indicated that this drift instability has a negligible effect on our experimental accuracy.

The normal line on which the $3.39-\mu$ laser oscillates is the very high gain $3 s_{2}-3 p_{4}$ transition. In order to study the $3 s_{2}-3 p_{2}$ transition, a 1-cm quartz absorption cell filled with methane to a pressure of 10 to 50 torr was introduced into the oscillator cavity. This completely quenched the $3 s_{2}-3 p_{4}$ oscillation and allowed the oscillation to take place on the $3 s_{2}-3 p_{2}$ line [6]. We passed the laser output through a Spex monochromator with a resolution of $0.2 \mathrm{~cm}^{-1}$ in order to determine the optimum pressure of methane needed.

A second tube of 4-mm bore and 110-cm length was also tested with the $J=1$ to $J=2$ line only.

\section{Results and Discussion}

First, gain measurements were taken for both transitions at very low-input intensity to measure the smallsignal inhomogeneous gain constants. Results gave a gain ratio of $\alpha\left(3 s_{2}-3 p_{4}\right) / \alpha\left(3 s_{2}-3 p_{2}\right)=2.7$ for discharge currents from 10 to $17 \mathrm{~mA}$. This is close to the theoretical ratio of line strengths [8], which is 2.55 .

Gain saturation data were next taken and fitted to theoretical expressions using the strong-signal theory for a linearly polarized signal at line center in zero magnetic field [4], [9]. The values of $\alpha$ obtained were 4.96/meter at a discharge current of $14 \mathrm{~mA}$ for the $J=1$ to $J=2$ line, and $1.76 /$ meter at a discharge current of $12 \mathrm{~mA}$ for the $J=1$ to $J=1$ line. Using the fact that the saturation curves depend on $a$, we deduced values of $a=0.3$ for both transitions in this manner. We use the condition $A+B=$ $a\left(\gamma_{a}+\gamma_{b}=2 \gamma_{a b}\right)$ in our calculations although this is not strictly true for the gas pressure used in our amplifier tube. Howerer the theory pertinent to these experiments is rather insensitive to the value of $\gamma_{b}$, and we can apply this condition with impunity. Thus, in later calculations we assume the decay rates of the $3 p_{2}$ and $3 p_{4}$ levels are equal.

In Figs. 2, 3, and 4 each experimental point represents an average from several successive measurements taken under the same conditions. With the values of $\alpha$ and $a$ determined from the gain saturation data, and $\omega_{+-}=3.94$ $\mathrm{MHz} / \mathrm{G}$, good fits to the experimental points of Figs. 2 and 3 were obtained for $A=0.05, B=0.25, k u=$ 165 MHz. Fig. 2 shows the traveling-wave magneticfield gain dip for the $J=1$ to $J=1$ line. The solid lines are obtained from (5a). The width of the dip depends on $A$, since it occurs because of the effects of coherent interactions via the Zeeman split upper sublevels. This gives a good method of determining the upper-state decay constant.

Fig. 3(a) shows the Faraday rotation versus magnetic field for various values of input-signal strength for the 


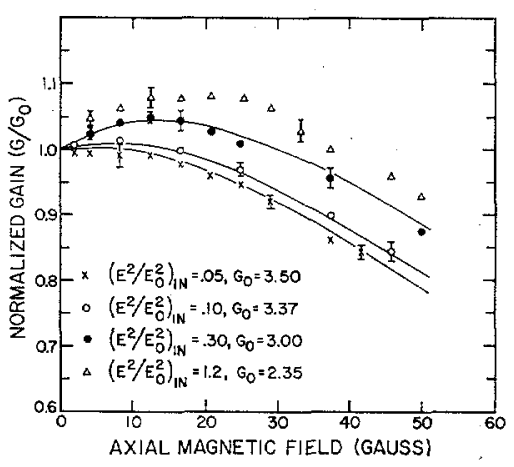

Fig. 2. Gain versus magnetic field for various strengths of linearly polarized 3.3912- $\mu(J=1 \rightarrow J=1)$ input signals, along with corresponding prediction of weak-field nonlinear theory (solid lines). Discharge current is $12 \mathrm{~mA}$. Values of atomic parameters are $a=\gamma_{a b} / k u=0.3, A=\gamma_{a} / 2 k u=0.05, k u=165 \mathrm{MHz}$, $\alpha=1.76$ meter $^{-1}, \omega_{+}{ }^{b}=3.94 \mathrm{MHz} / \mathrm{G}$. Representative error brackets are included.

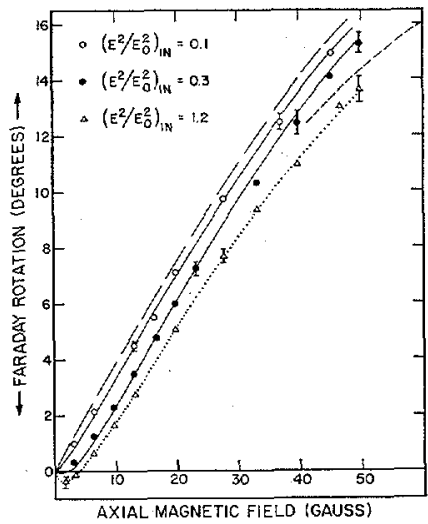

(a)

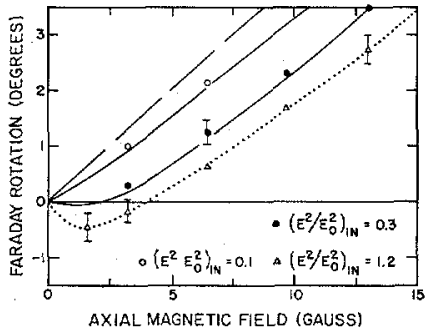

(b)

Fig. 3. (a) Faraday rotation for various values of $3.3912-\mu$ ( $J=$ $1 \rightarrow J=1$ ) input signals, along with corresponding predictions of linear theory (long dash), and weak-field nonlinear theory (solid), using the same values for the discharge parameters as given in Fig. 2. Representative error brackets are included. Note negative values of rotation at low values of magnetic field and high signal strength. Short dash curve is approximate strongsignal theory for high-magnetic fields (6). The dotted curve is merely a line joining the experimental points. (b) This is an enlargement of the weak magnetic field region of (a).

$J=1$ to $J=1$ line. Fig. $3(\mathrm{~b})$ is an enlargement of the weak magnetic field region of Fig. 3(a). The long dashed curve is the unsaturated Faraday rotation obtained by assuming the polarization of the medium to be linearly dependent on signal strength:

$$
\Phi_{l}=\alpha z \operatorname{Im} W(y) .
$$

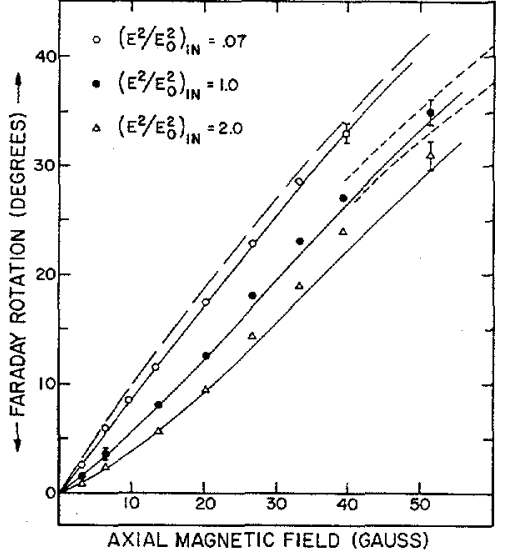

Fig. 4. Faraday rotation for various values of $3.3923-\mu(J=1 \rightarrow$ $J=2$ ) input signals, with corresponding predictions of linear theory (long dash), and strong-field nonlinear theory; equation (7), solid. Discharge current is $14 \mathrm{~mA} ; a=0.3, B=0.3, A \simeq 0$, $\alpha=4.96$ meter $^{-1}, \omega_{+}{ }^{b}=3.33 \mathrm{MHz} / \mathrm{G}$. Short dash curve is approximate strong-signal theory for large magnetic fields.

The solid curves are theoretical lines using (5b). We see that the weak-signal theory is quite accurate in its region of validity. We observe a reversal of the Faraday rotation at small magnetic fields when $I_{\text {in }}=1.2$ as predicted in Section II, although the perturbational equations no longer hold in the quantitative sense. (The dotted curve is merely a line joining the experimental points.) Presumably larger signals would produce a large dip in the rotation; thus our theoretical predictions are qualitatively accurate for large signals.

The short dashed line is a theoretical curve using (6) for $I_{\text {in }}=1.2$. Since the value of $y$ at 50 gauss is 0.54 , the condition of validity for (6) is not quite satisfied. The effects of the coherent interactions are not negligible; thus we expect the experimental points to be a little lower than the dashed curve.

Fig. 4 shows the Faraday rotation versus magnetic field for the $J=1$ to $J=2$ line. The long dashed curve is once again the unsaturated rotation. Comparison of Figs. 3 and 4 supports our argument that the effects of the coherent interaction terms are much stronger for $J=1$ to $J=1$ than for $J=1$ to $J=2$. There is no reversal of the rotation for the $J=1$ to $J=2$ line and the nonlinear effects at low magnetic fields are much smaller. The use of the perturbational results [see (5)] was not possible. The gain for $I_{\text {in }}=0.07$ was 26 , which means that near the output the signal strength was too large for the weaksignal theory even for this case, and the results indicate only a slight decrease in Faraday rotation from that predicted by the linear theory. The short dashed lines were obtained from (6). The observation made for the $J=1$ to $J=1$ line holds here too. The solid lines were obtained from (7). The approximation made in this expression is that $B=a=0.3$. Actually $B$ is smaller than this value; (7) therefore predicts too much saturation and we expect the experimental points to be slightly higher than the 
solid curves. This is in fact the case, although the overall fit is satisfactory.

Results obtained on the long tube were qualitatively very similar to those shown in Fig. 4 . Since the magnetic field on this tube was somewhat inhomogeneous, no attempt was made to fit this data to theoretical expressions. The gain of this tube was considerably higher and much larger Faraday rotations were observed. Rotations as high as $105^{\circ}$ were seen, and saturation effects were considerable.

\section{Conclusions}

We have investigated the nonlinear behavior of gain and Faraday rotation of a linearly polarized optical signal in a gaseous amplifying medium subject to an axial magnetic field. Good agreement between theory and experiment were found. It may be appropriate to note at this point that when the amplifier operates in the nonlinear region the Faraday rotation cannot, in general, be obtained from the gain using the dispersion (Kramers-Kroenig) relations, but must be calculated from the rigorous theoretical expressions [10].

We have also found useful values of linewidths and decay constants in the $3.39-\mu$ region. The parameter $A$ was given various values in applying (5a) and (5b) to the appropriate experimental points in Figs. 2 and 3 . The resulting fits allowed us to determine confidence limits for the value of $\gamma_{a}$. For the discharge tube tested the results are $(k u / 2 \pi)=165 \mathrm{MHz},\left(\gamma_{a b} / 2 \pi\right)=49.5 \mathrm{MHz}$, and $\left(\gamma_{a} / 2 \pi\right)=16.0 \pm 3.0 \mathrm{MHz}$. This value for $\gamma_{a}$ is larger than the corresponding quantity obtained from the laser-excited Hanle effect experiment by Decomps and Dumont [11]. Their value was reported to be about 10.3 $\mathrm{MHz}$.

Tomlinson and Fork [2] made allowances for pressure effects on the laser medium by relaxing the condition $\gamma_{a}+\gamma_{b}=2 \gamma_{a b}$ in their analysis. However this was not necessary in order to match theoretical predictions to experimental observations in our case. This is partially due to the fact that the phenomena that we have studied give more sensitive indications of the value of $\gamma_{a}$ than that of $\gamma_{b}$. We can assume $\gamma_{a}+\gamma_{b} \leq 2 \gamma_{a b}$ and obtain good fits to the experimental points for a fairly large range of values of $\gamma_{b}$. (The range of values for $\gamma_{a}$ necessary to obtain good fits remains virtually unchanged however.) The reason that this was not necessary is also due to the fact that the gas pressure in our discharge tube was roughly half the gas pressure used in their experiments; thus reduced pressure effects should be expected.

In the theory that is presented in this paper the quantities $\gamma_{a}$ and $\gamma_{b}$ are each the sum of the spontaneous decay rate and the decay rate due to excitation transfer collisions with neighboring atoms that induce transitions to the ground level. By generalizing the gas laser theory of D'yakonov and Perel' [12] to include collision effects on the Zeeman sublevel populations [13], it can be shown that the quantity $\gamma_{a}$, which we have experimentally measured by studying the width of the coherent interaction dip, is equal to the quadrupole moment decay rate of the upper $\left(3 s_{2}\right)$ level,

$$
\Gamma_{a}^{(2)}=\Gamma_{a}+\left(\Gamma_{a}^{(2)}\right)_{\mathrm{col1}}
$$

where $\Gamma_{a}$ is the decay rate due to spontaneous emission and excitation transfer collisions (the monopole moment decay rate), and the added term is due to Zeeman sublevelmixing collisions. Recent investigations [14], [15] have shown that for the gas mixture and pressure used in our amplifier tube, $\Gamma_{a}^{(2)}$ is 20 to 30 percent larger than $\Gamma_{a}$; hence, the distinction between the quadrupole and monopole moment decay rates must be made.

\section{ACKNOWLEDGMENT}

The authors would like to thank R. L. Abrams for suggesting the use of the $3.3912-\mu J=1$ to $J=1$ line. The assistance of M. Sargent, III, with computer programs is also gratefully acknowledged.

\section{RFFERENCES}

[1] M. Sargent, III, W. E. Lamb, Jr., and R. L. Fork, "Theory of a Zeeman laser, I and II," Phys. Rev., vol. 164, pp. 436-449 and 450-465, December 1967.

[2] W. J. Tomlinson and R. L. Fork, "Properties of gaseous optical masers in weak axial magnetic fields," Phys. Rev., vol. 164, pp. $466-484$, December 1967.

[3] A. Dienes, "Theory of nonlinear effects in a gas laser amplifier; I-Weak signals," Phys. Rev., vol. 174, pp. 400-414, October 1968; also Ph.D. dissertation, California Institute of Technology, Pasadena, Calif., Sci. Rept. 6, AF49(638)-1322, 1967.

[4] - "Theory of nonlinear effects in a gas laser amplifier; II-S'trong signals," Phys. Rev., vol. 174, pp. 414-423, October 1968; also Ph.D. dissertation, California Institute of Technology, Pasadena, Calif., Sci. Rept. 6, AF49(638)-1322, 1967.

[5] C. E. Moore, NBS circ. $467,1949$.

[6] C. Bradley Moore "Gas-laser frequency selection by molecular absorption," Appl. Opt., vol. 4, pp. 252-253, February 1965.

[7] M. Abramowitz and I. A. Stegun, Eds., Handbook of Mathematical Functions. Appl. Math. Ser. 55, NBS, Washington, D. C., 1964, pp. 297-304, 325-328.

[8] P. W. Murphy, "Transition probabilities in the spectra of NeI, ArI, and KrI," J. Opt. Soc. Am., vol. 58, pp. 1200-1208, September 1968. The wavelength values in this reference are given in air, whereas the values in the text are in vacuum.

[9] A. Dienes, "Polarization-dependent gain saturation and nonlinearity-induced anisotropy in the $3.39-\mu \mathrm{He}-\mathrm{Ne}$ laser amplifier," IEEE J. Quantum Electronics, vol. QE-5, pp. 162-166, March 1969.

[10] A. M. Portis, "Electronic structure of F-centers: saturation of the electron-spin resonance," Phys. Rev., vol. 91, pp. 1071-1078, September 1953.

[11] B. Decomps and M. Dumont, "Polarization of the fluorescent light of Ne atoms interacting with a laser beam; measurement of several relaxation times," IEEE J. Quantum Electronics, vol. QE-4, pp. 916-922, November 1968

[12] M. I. D'yakonov and V. I. Perel', Opt. i Speltroskopia, vol. 20, p. 472, 1966 (transl.), "On the theory of a gas laser in a magnetic field," Opt. Specty., vol. 20, pp. 257-261, 1966.

[13] W. J. Tomlinson and R. L. Fork, "Use of nonlinear optical interactions to measure relaxation rates of atomic multipole moments," Phys. Rev. Letters, vol. 20, pp. 647-649, March 25, 1968.

[14] C. H. Wang, W. J. Tomlinson, and R. T. George, Jr., "Collision induced anisotropic relaxation in a gas laser," Phys. Rev., vol. 181, pp. 125-136, May 1969. 